\title{
INVESTIGACIONES
}

\section{Desempeño ortográfico de estudiantes chilenos: claves para la enseñanza de la ortografía*}

\author{
Orthographic performance in Chilean students: Keys for teaching spelling
}

\author{
Carmen Sotomayor, ${ }^{a}$ Natalia Ávila, ${ }^{a b}$ Percy Bedwell, ${ }^{c}$ \\ Ana Domínguez, ${ }^{\text {cd }}$ Gabriela Gómez, ${ }^{a e}$ Elvira Jéldrezaf \\ aCentro de Investigación Avanzada en Educación, Universidad de Chile \\ Telf.: (56) 22978 2762. Correo electrónico: csotomayor@ ciae.uchile.cl \\ bTelf.: (56) 22978 2762. Correo electrónico: natalia.avila@ ciae.uchile.cl \\ 'Fundación Educacional Arauco \\ Telf.: (56) 224994800. Correo electrónico: Percy.Bedwell@ arauco.cl \\ dTelf.: (56) 224994800. Correo electrónico: AnaMaria.Dominguez@ arauco.cl \\ eTelf.: (56) 22978 2762. Correo electrónico: gabriela.gomez@ ciae.uchile.cl \\ fTelf.: (56) 22978 2762. Correo electrónico: elvira.jeldrez@ ciae.uchile.cl
}

\begin{abstract}
RESUMEN
Aunque posee una gran importancia en diversos aspectos de la escritura, la ortografía es un tema poco abordado en la investigación actual. Este artículo propone presentar un panorama exhaustivo, reciente y representativo del desempeño ortográfico de niños chilenos, para ser utilizado como insumo para su enseñanza. Para ello, se analizó una muestra estratificada proporcional desde una muestra representativa del país tomada en el piloto SIMCE de escritura del año 2008 en cuarto grado. Los resultados fueron luego interpretados desde perspectivas cognitivas, comunicativas y didácticas de la ortografía. Entre los principales hallazgos, destaca un alto porcentaje de palabras correctamente escritas, la concentración de los errores en unos pocos tipos y un mejor desempeño en el género carta de solicitud por sobre el cuento y la noticia. En conjunto, se sugiere focalizar la instrucción en los errores encontrados e insertarla en el desarrollo de textos en situaciones comunicativas.
\end{abstract}

Palabras clave: escritura, educación básica, ortografía.

\section{ABSTRACT}

In spite of being important for several aspects of writing, orthography has been scarcely discussed in current research. This article presents a comprehensive, current and representative picture of the orthographic performance of fourth-grade Chilean students, in order to use this data to inform teaching. A stratified sample taken from the representative sample of the national assessment SIMCE writing pilot of 2008 was analyzed. The results were later interpreted in terms of cognitive, communicative and didactics approaches. Among the main findings, a high percentage of correctly written words and a better performance in the genre of the formal letter stand out significantly over the genres short story and news report. In sum, it is suggested to focus instruction on the mistakes found, rather than teaching norms; and to embed this instruction within experiences of writing in communicative situations.

Key words: writing, primary education, spelling.

Se agradece financiamiento de Fondo Basal FB003 de Conicyt. 


\section{INTRODUCCIÓN}

La ortografía es un tema poco abordado en los estudios recientes sobre escritura escolar, a pesar de que cumple un importante rol en los procesos de transcripción, legibilidad y comunicación (Camps et al., 2007). Entre los pocos estudios actuales, destacan en Latinoamérica, el Segundo Estudio Regional Comparativo y Explicativo realizado por la Unesco (Atorresi et al., 2010), que encontró que el promedio de los 15 países participantes es un error de ortografía cada diez palabras en sexto grado. Los resultados de los estudiantes chilenos que participaron en este estudio no se diferencian significativamente de la muestra total. En el Tercer Estudio Regional Comparativo y Explicativo (Flotts et al., 2015), se optó por un enfoque diferente que evaluó la ortografía dentro de una rúbrica de "Convenciones de legibilidad", que agrupó los desempeños en ortografía literal, segmentación de palabras y usos básicos de la puntuación. A nivel regional, 38,2\% de los niños de Tercer grado se ubicaron en el nivel IV, el máximo posible, que supone ausencia de errores en el uso del punto seguido y la coma enumerativa, la totalidad de palabras segmentadas correctamente y ningún error de ortografía en que se asocie un grafema incorrectamente a un sonido, es decir, ningún error que no respete la fonología de las palabras. En Chile, un 50,6\% de los niños alcanzó este mismo nivel. En el caso de sexto grado, $56,1 \%$ de los niños en la región se ubicaron en el nivel IV, que supone uso correcto del punto seguido, final y coma enumerativa, la totalidad de las palabras segmentadas correctamente y hasta un error de ortografía literal por texto. El 66,4\% de los estudiantes chilenos alcanzaron este nivel.

En los países de la región, destaca un estudio brasileño de 2012, que analiza el rol del conocimiento ortográfico en el desempeño de niños de cuarto grado. Los niños con mejor desempeño cometían errores, pero estos no alteraban la fonología de las palabras (Dos Santos \& Barrera, 2012).

Por su parte, en un estudio realizado en México, Backhoff y otros (2008) mostraron que los estudiantes escriben incorrectamente un $23 \%$ de las palabras en tercero y un $17 \%$ en sexto grado, y que el Coeficiente de Error Ortográfico (CEO, calculado sumando el número de errores ortográficos que un alumno comete por cada 100 palabras escritas) es de 31 y 18 puntos, respectivamente, para ambos cursos. Se evaluaron 27 tipos de errores ortográficos y el más frecuente en ambos cursos fue el de acentuación, el que se presentaba en casi la totalidad de los alumnos. Al analizar este problema según la variable de sexo, se encontró que las mujeres presentaban un mejor desempeño. Otros problemas ortográficos frecuentes encontrados en este estudio fueron el uso incorrecto de $\mathrm{b} / \mathrm{v}$, de mayúsculas y de s/c/z, para ambos cursos. Existen muy pocos estudios sistemáticos en América Latina además de los hasta acá reportados y estos se remontan a inicios de la década de los 80 . Entre sus resultados ya se encontraba el mejor desempeño de las mujeres y una mayor incidencia de errores en palabras comunes (Tuana, 1980; Tuana, Carbonell \& Lluch, 1980).

Por último, el estudio realizado por Sotomayor y otros (2013) constituye el principal antecedente chileno. En este se analizaron narraciones escritas por alumnos de tercero, quinto y séptimo grado de escuelas de nivel socioeconómico bajo en un conjunto de escuelas de la zona sur de Chile, y se concluyó que los problemas ortográficos más frecuentes eran la carencia de tildes, el uso erróneo de grafías (b/v; s/c/z; h), la hiposegmentación y la omisión o cambio de sílabas o letras. Además, se encontró que los problemas ortográficos se concentraban en el $17 \%$ de las palabras escritas y que, en el caso de la mayoría de los problemas estudiados, los alumnos de quinto y séptimo grado presentaron menos errores 
que los de tercero. Molina (2012) obtuvo similares resultados e identificó como problema más frecuente la tildación en las palabras agudas y en el hiato. Además, observó que los problemas de omisión o cambio de grafías y de hiposegmentación se daban frecuentemente en los escritos de alumnos de tercer grado.

Además de tratarse de un tema escasamente investigado, en la mayoría de los estudios empíricos sobre ortografía en edad escolar se omiten factores relacionados con el contexto de producción textual u otras variables de la situación que resultan relevantes para su didáctica. Tampoco existe hasta ahora ningún estudio sobre el desempeño ortográfico que sea representativo de nuestro país, lo que dificulta el acceso a información útil para la implementación de acciones didácticas al respecto. En otras palabras, hay escasa información acerca de qué saben los niños chilenos sobre ortografía y, por tanto, la toma de decisiones sobre qué y cómo se debería enseñar no suele estar informada por datos, sino que por percepciones o representaciones, las que suelen subestimar el desempeño ortográfico de los estudiantes en particular, y percibir una crisis de la escritura en general (Gee, 2004).

Con el fin de aportar datos en esta dirección, el presente estudio busca describir de manera exhaustiva la calidad del desempeño ortográfico en una sub-muestra estratificada proporcional de 269 textos escritos por 101 alumnos de cuarto grado, tomada desde una muestra representativa del país. En esta caracterización se establecen relaciones con variables textuales, como el número de palabras de cada escrito y su calidad global; variables del contexto situacional, como el género que se escribe; y variables sociales, tales como el nivel socioeconómico y el sexo de los estudiantes.

De esta manera, los resultados del presente estudio buscan ofrecer información concreta a la comunidad educativa sobre el estado actual del desempeño ortográfico en Chile, pero también -y a partir de esta evidencia- ofrecer algunas ideas acerca de cuáles deberían ser los principales focos de la enseñanza.

\section{MARCO TEÓRICO}

\subsection{LA ORTOGRAFÍA DESDE UNA PERSPECTIVA COGNITIVA Y DE DESARROLLO}

La escritura es una competencia básica y fundamental para el desarrollo del aprendizaje, la comunicación y la participación en la sociedad. Su adquisición implica un proceso largo y complejo, pues involucra múltiples habilidades en forma simultánea, como por ejemplo, la capacidad de utilizar el vocabulario, la estructura textual o la ortografía (Smith-Lock, Nickels \& Mortensen, 2009). La integración de estas habilidades implica una alta demanda para la memoria de trabajo (Berninger et al., 1992). Esta memoria es limitada, ya que depende en gran medida del grado de automatización de las tareas implicadas (Sánchez, Moyano \& Borzone, 2011). En este sentido, la ortografía debiera llegar a automatizarse como parte de los procesos de codificación, lo cual permitiría utilizar la memoria de trabajo en otros procesos como la planificación o la revisión de la escritura (McCutchen, 2011).

En la enseñanza de la ortografía es fundamental considerar las distintas etapas del desarrollo en que se encuentran los alumnos (Camps et al., 2007). En esta perspectiva, muchos de los errores tienen sentido, pues son parte del proceso de aprendizaje de las regularidades del código gráfico. No obstante, el dominio de habilidades cognitivas 
inferiores en las etapas iniciales de la instrucción formal de la escritura, entre las que se incluye la transcripción ortográfica, es un predictor de la adquisición del código escrito y puede influir en los procesos de transcripción y en la adquisición de habilidades cognitivas superiores de los procesos de composición en etapas futuras del desarrollo (Berninger et al., 1992). Por ello, es importante que dicho aprendizaje se promueva de manera intencionada. Para lograr este aprendizaje es fundamental considerar a los alumnos como sujetos pensantes (Ferreiro, 2007), apelando a su inteligencia de modo de estimular y potenciar una reflexión en torno a las normas ortográficas que habilite su adquisición progresiva.

\subsection{LA ORTOGRAFÍA DESDE UNA PERSPECTIVA COMUNICATIVA Y SOCIAL}

La ortografía cumple un rol social e identitario al mantener la unidad de quienes hablan una misma lengua y hace posible que un texto pueda resultar legible para una vasta comunidad de hablantes, como es el caso de los hablantes de español (Camps et al., 2007; Real Academia de la Lengua Española, 2010). Por otra parte, la incorrección ortográfica en las prácticas letradas más centrales y prestigiosas, como la escritura escolar, académica y laboral o los foros públicos, tiene una connotación negativa. Las creencias sociales sobre la ortografía promueven una expectativa de corrección en determinados ámbitos, que alimenta prejuicios hacia quienes escriben sin apego a la norma, lo que incrementa la importancia de enseñarla a los estudiantes para que puedan insertarse y participar de estos espacios sociales (Campbell Wilcox, Yagelski \& Yu, 2014; Sebba, 2007).

Comprender la ortografía desde una perspectiva comunicativa implica entender la escritura como un proceso situado en el que los textos son escritos para un lector que espera el uso de las convenciones para poder leer el escrito. Esta perspectiva puede representar un importante factor de motivación a la hora de desarrollar el desempeño ortográfico de los niños.

Sin embargo, la adquisición de la ortografía no es tarea fácil, porque se trata de reglas arbitrarias basadas en un conjunto de convenciones que adopta una comunidad de hablantes en un momento dado. Tal como lo indican Camps et al. (2007): "El conjunto de normas que regula el código ortográfico se establece sobre la base de un acuerdo aceptado por los usuarios de dicha lengua" (p. 17). Por otra parte, la relación entre lengua escrita y hablada no es siempre unívoca ni transparente en los sistemas de escritura alfabéticos, dado precisamente el carácter convencional del código gráfico (Blanche-Benveniste, 1998). Esto ocurre con algunos sonidos cuya representación gráfica en el español de Chile puede tener varias posibilidades, como es el caso de /k/ (grafías q, c, k); /s/ (grafías s, c, z) o /b/ (grafías b y v), entre otras. Los principales problemas en la adquisición de la ortografía literal, por lo tanto, tenderán a presentarse en estos casos de desajuste entre el código gráfico y la oralidad.

En síntesis, desde un punto de vista cognitivo y del desarrollo, el aprendizaje de la ortografía es relevante en la adquisición del código escrito en los niños. Junto a los procesos de composición, el dominio del código constituye la base de la producción escrita. Desde un punto de vista comunicativo, el conocimiento ortográfico es indispensable para la comunicabilidad de los escritos y para la comunicación entre comunidades que comparten una misma lengua. Desde un punto de vista social, la ortografía importa al público general, y se generan expectativas de desempeño y prejuicios asociados al desapego a la norma. Sin embargo, su carácter convencional imprime diversas dificultades para su aprendizaje, lo que hace necesaria su mediación explícita en aula, como veremos a continuación. 


\subsection{LA ORTOGRAFÍA DESDE UNA PERSPECTIVA DIDÁCTICA}

Tal como señalan diversos autores, la enseñanza tradicional de reglas ortográficas descontextualizadas es muchas veces infructuosa, ya sea porque no se focaliza en los errores que los estudiantes efectivamente cometen (Campbell Wilcox et al., 2014) o porque obstaculizan los procesos de reflexión y elaboración de regularidades en torno al error (Morales \& Hernández, 2004).

Aunque no hay pleno consenso sobre el significado del error, y este puede ser considerado ya sea como parte de la construcción del conocimiento ortográfico o como una desviación de la norma, este debe utilizarse en el aula para la enseñanza de la ortografía, pues representa un insumo valioso que expande la utilidad de la enseñanza de reglas y promueve su aplicación. Quienes se inclinan por estas propuestas didácticas señalan que las dificultades ortográficas deben caracterizarse guiando a los alumnos para que puedan descubrirlas (Díaz, 2010). En esta línea, Sánchez (2010) señala que los errores se pueden abordar potenciando el trabajo entre los alumnos con distintas estrategias, tales como la revisión y edición en grupos, el intercambio de escritos, la corrección colaborativa o el registro de las dificultades más frecuentes. En todos estos casos, el foco de la enseñanza debe estar puesto en reflexionar respecto de la ortografía, especialmente, en situaciones concretas de producción. La reflexión acerca de los patrones ortográficos está también presente en las propuestas didácticas de Kaufman (2005) y Camps et al. (2007), y constituyen también uno de los principales resultados sugeridos por los datos de investigación de Dos Santos y Barrera (2012).

Un último factor clave en la enseñanza de la ortografía es considerarla dentro del proceso de escritura que debe enmarcar las acciones didácticas en situaciones comunicativas: "El niño sentirá la necesidad de la ortografía solo si siente la necesidad de escribir" (Camps et al., 2007, p. 42). Del mismo modo, una enseñanza contextualizada y enfocada permite repensar la incidencia del error. Ruiz (2009) distingue las equivocaciones (errores azarosos), que ocurren probablemente debido a la falta de atención, de los errores propiamente tal (errores sistemáticos), debidos al desconocimiento de la forma normalizada. El trabajo con adecuadas estrategias de revisión puede reducir drásticamente la incidencia de los errores azarosos y facilitar la enseñanza focalizada de los errores sistemáticos.

En síntesis, la investigación indica que la ortografía debe enseñarse en la escuela de manera explícita e intencionada, en el contexto de la práctica de la escritura, esto es, en textos y situaciones de escritura concretas. Los esfuerzos y tiempos de enseñanza, no obstante, deben ser acotados y focalizarse en las necesidades, ya que la ortografía es solo una entre otras habilidades, muchas de ellas más complejas, requeridas para la escritura. Como bien lo subrayan Camps et al. (2007): "Enseñar a escribir no es enseñar ortografía, aunque para comunicarnos por escrito se necesita conocer la norma ortográfica de la lengua empleada" (p. 41). Condemarín y Medina (2000), citando al Grupo EVA, señalan en el mismo sentido: "La ortografía ocupa un lugar importante en el acto de escribir, pero no ocupa todo el lugar" (p. 52). Es por esto que una descripción exhaustiva y actualizada de los problemas ortográficos más recurrentes de los niños del país puede aportar datos concretos para optimizar la instrucción en esta área. 


\section{METODOLOGÍA}

\subsection{MUESTRA}

El objetivo del estudio es caracterizar el desempeño ortográfico de los alumnos de cuarto grado en Chile, estableciendo relaciones con algunas variables textuales (número de palabras, calidad global de la escritura), del contexto situacional de los textos (género escrito), y del contexto social de los escritores (sexo y nivel socio-económico).

Con este propósito, se trabajó con una muestra de 101 alumnos que realizaron 269 escritos obtenidos de la prueba piloto del Sistema de Medición de la Calidad de la Educación (SIMCE) de escritura realizada el año 2008. Esta prueba fue aplicada a una muestra representativa a nivel nacional (Ministerio de Educación de Chile, 2009). Para la selección de la muestra de ortografía se consideraron los mismos criterios de selección de la muestra nacional, a saber, nivel socioeconómico y sexo. Cada niño debía escribir tres textos correspondientes a diferentes géneros (cuento, carta de solicitud y noticia), sin embargo, algunos escribieron solamente uno o dos de los textos solicitados. De los 101 alumnos de la muestra, solo 77 realizaron las tres tareas de escritura. De este modo, la muestra total quedó conformada por 269 textos, aunque para el cálculo de correlaciones solo se consideraron 231 textos escritos por 77 alumnos (Tablas 1a, 1b).

Tabla 1a. Composición de la muestra

\begin{tabular}{|l|c|c|c|c|}
\hline \multicolumn{5}{|c|}{ NSE } \\
\hline Género textual & Bajo/Medio bajo & Medio & Medio Alto/Alto & Total \\
\hline Cuento & 34 & 33 & 23 & 90 \\
\hline Carta & 34 & 33 & 23 & 90 \\
\hline Noticia & 34 & 32 & 23 & 89 \\
\hline Total & 102 & 98 & 69 & 269 \\
\hline
\end{tabular}

Tabla 1b. Composición de la muestra

\begin{tabular}{|l|c|c|c|}
\hline \multicolumn{4}{|c|}{ Sexo } \\
\hline Género textual & Masc. & Fem. & Total \\
\hline Cuento & 45 & 45 & 90 \\
\hline Carta & 45 & 45 & 90 \\
\hline Noticia & 44 & 45 & 89 \\
\hline Total & 134 & 135 & 269 \\
\hline
\end{tabular}

Fuente: elaboración propia. 


\subsection{ANÁLISIS DE PROBLEMAS ORTOGRÁFICOS}

Los 269 textos fueron transcritos en procesador de textos con ortografía correcta para facilitar la legibilidad, lo que permitió el cálculo de totales de palabras escritas y de palabras con error. A continuación, se clasificó cada error encontrado en los textos originales en una taxonomía de problemas ortográficos tomada del estudio sobre ortografía escolar realizado por el Instituto Nacional para la Evaluación de México (INEE) (Backhoff et al., 2008) e ilustrada con ejemplos del estudio de caracterización de problemas ortográficos de Sotomayor et al. (2013). Con estos antecedentes fue posible distinguir entre 26 diferentes tipos de problemas ortográficos, los que se presentan en la Tabla 2.

Tabla 2. Tipos de problemas ortográficos

\begin{tabular}{|c|l|l|}
\hline Código & \multicolumn{1}{|c|}{ Tipo de error } & \multicolumn{1}{c|}{ Ejemplo de error } \\
\hline 1 & Adición de acentos & Adición incorrecta de tildes \\
\hline 2 & Carencia de acentos & Omisión de tildes \\
\hline 3 & Acentuación incorrecta en pretérito (on) & Salierón, corrierón \\
\hline 4 & Uso de mayúscula & Planeta marte/planeta Marte \\
\hline 5 & Omisión de letras o sílabas & Pa/para \\
\hline 6 & Adición de letras o sílabas & Dijistes/dijiste, conriendo/corriendo \\
\hline 7 & Hiposegmentación & Lacasa \\
\hline 8 & Hipersegmentación & Ca sa \\
\hline 9 & Palabras homófonas & Vez/ves, ahí/hay/a y/ay (no hai o ai) \\
\hline 10 & Confusión B/D y V/D & Hadía/había \\
\hline 11 & Uso de B/V & Jugaba/jugaba, octavo/octavo \\
\hline 12 & Uso de MB y MP & Canpo/campo \\
\hline 13 & Uso de NV & Conversar/conversar \\
\hline 14 & Uso de G y J & Gente/jente, segia/seguía \\
\hline 15 & Uso de diéresis & Omisión, adición y reemplazo de diéresis we/güe \\
\hline 16 & Uso de H & Uevo/huevo \\
\hline 17 & Uso de LL/Y & Llo/yo, yegaron/llegaron \\
\hline 18 & Uso de Q/C/K & Por ce/por que \\
\hline 19 & Uso de R/RR & Rregresaron/regresaron \\
\hline 20 & Uso de S/C/Z & Serdo/cerdo \\
\hline 21 & Uso de X & Axión/acción \\
\hline 22 & Confusión M/N/Ñ & Aninales/animales \\
\hline 23 & Confusión E/I & Caldeado/caldiado \\
\hline 24 & Confusión LL/N & Cumpleallos/cumpleaños \\
\hline 25 & Confusión Y/LL/CH & Muyo/mucho \\
\hline 26 & Otros & \\
\hline & & \\
\hline
\end{tabular}


Para medir el uso del dominio ortográfico y tener un control en relación con el número de palabras escritas, se utilizó el coeficiente de Bolet (1999, Cit. en Backhoff et al., 2008), que se obtuvo al multiplicar los errores de cada alumno por cien y luego dividir este producto por el número de palabras escritas en el texto. Para realizar este análisis, y con el objetivo de poder comparar estadísticamente según los géneros escritos, se trabajó solamente con la muestra de alumnos que hubiera escrito los tres tipos de textos $(\mathrm{n}=77)$ lo que conforma un total de 231 textos. Con este índice se llevaron a cabo análisis comparativos y de correlaciones.

Para la presentación de los resultados, se realizaron análisis descriptivos, calculando el promedio y medidas de dispersión (distribuciones y desviación estándar).

Por último, para estimar la incidencia de equivocaciones (errores azarosos), que no denotan falta de competencia, se contabilizaron, por niño, las veces en que una palabra con error fue escrita de modo correcto.

\subsection{CONSIDERACIÓN DE FACTORES TEXTUALES, SITUACIONALES Y SOCIALES}

Para la detección de diferencias de medias entre los coeficientes de error ortográfico, se utilizó la ANOVA de un factor con medias repetidas para comparar los tres promedios y posteriormente se utilizó el test de Bonferroni para la comparación de pares, en tanto que para medir la relación lineal entre las distintas variables, se utilizó el Coeficiente de correlación de Pearson.

Estos análisis fueron utilizados para indagar relaciones entre el desempeño ortográfico y otros aspectos del texto, la situación comunicativa o el contexto social, que pueden brindar datos útiles para informar la enseñanza. Las variables consideradas para este análisis, además de los tres géneros escritos, fueron:

- Número de palabras: número total de palabras escritas en el texto. Para esto se consideró su transcripción en ortografía correcta.

- Calidad del texto: para obtener una medida referencial de la calidad de la escritura, se construyeron índices para cada tipo de texto, para lo cual se desarrollaron análisis de componente principal de distintas dimensiones evaluadas con una alta consistencia interna $(\alpha>0,79)$, considerando las dimensiones significativamente correlacionadas en cada caso (coherencia, cohesión, estructura y evaluación global en los cuentos; adecuación, coherencia, estructura y evaluación global en las cartas; adecuación coherencia y evaluación global en noticias). Los índices fueron estandarizados en puntaje T para tener una media de 50 y una desviación estándar de 10 puntos.

- Nivel Socio-económico (NSE): caracterización demográfica del nivel socioeconómico de los estudiantes participantes provisto por el SIMCE.

- $\quad$ Sexo: hombres y mujeres. 


\section{RESULTADOS}

\subsection{ANÁLISIS DESCRIPTIVO}

\subsection{1. $\quad$ Número de palabras escritas y porcentaje de palabras correctas}

Para este análisis se tomó el total de escritos disponibles (269) considerando los tres géneros textuales, lo cual se presenta en la Tabla 3. Cabe destacar que el cuento correspondía a una narración elicitada desde una fotografía; la carta, a una solicitud dirigida al director(a) del establecimiento educacional para un tema específico; y la noticia, a un reporte de un evento en un diario local, reforzado por una fotografía.

Tabla 3. Cantidad de palabras escritas en total y porcentaje de palabras escritas correctamente

\begin{tabular}{|l|c|c|c|c|c|c|c|c|}
\hline \multirow{2}{*}{} & \multicolumn{2}{|c|}{$\begin{array}{c}\text { Cuento } \\
(\mathrm{n}=90)\end{array}$} & \multicolumn{2}{c|}{$\begin{array}{c}\text { Carta } \\
(\mathrm{n}=90)\end{array}$} & \multicolumn{2}{c|}{$\begin{array}{c}\text { Noticia } \\
(\mathrm{n}=89)\end{array}$} & \multicolumn{2}{c|}{$\begin{array}{c}\text { Total } \\
(\mathrm{n}=269)\end{array}$} \\
\cline { 2 - 10 } & Media & DE & Media & DE & Media & DE & Media & DE \\
\hline Total de palabras escritas & 101,2 & 31,6 & 50,1 & 23,3 & 46,7 & 25,5 & 66,1 & 36,8 \\
\hline $\begin{array}{l}\text { Porcentaje de palabras } \\
\text { escritas correctamente }\end{array}$ & $90,5 \%$ & 3,8 & $92,7 \%$ & 3,9 & $90,6 \%$ & 4,3 & 91,3 & 4,1 \\
\hline
\end{tabular}

Fuente: elaboración propia.

Los alumnos evaluados escriben en promedio 66 palabras por texto, sin embargo, es posible ver que esta situación varía según el género escrito. Cuando producen cuentos, utilizan un mayor número de palabras, el doble que cuando escriben una carta o noticia y entre estos dos últimos géneros no se aprecian mayores diferencias en cuanto al número de palabras.

Cabe destacar que la gran mayoría de las palabras (más del 90\%) están escritas correctamente y al analizar por género escrito, no se aprecian mayores diferencias en estos porcentajes.

\subsubsection{Errores ortográficos más frecuentes}

En total, considerando los tres géneros textuales analizados, los estudiantes presentaron 3.136 errores. La Tabla 4 muestra el porcentaje de errores por tipo. Los errores más frecuentes son carencia de tildes, omisión de letras o sílabas, uso erróneo de grafías b/v, h, s/c/z e hiposegmentación y todos estos concentran el $84 \%$ del total de los errores, mientras que los otros veinte tipos concentran el $16 \%$.

El error ortográfico más recurrente es la carencia de tilde $(49,2 \%)$. En segundo lugar, se puede identificar un grupo de errores relacionados con la omisión de letras o sílabas y el uso erróneo de grafías (b/v, h, s/c/z), seguidos de los errores de hiposegmentación. Este porcentaje se calculó al considerar como cien por ciento la suma total de los errores presentados por los estudiantes. 
Tabla 4. Número y porcentaje de errores ortográficos

\begin{tabular}{|l|c|c|c|c|c|c|c|c|}
\hline \multirow{2}{*}{ Tipos de errores } & \multicolumn{6}{|c|}{ Género textual } & \multicolumn{2}{|c|}{ Total } \\
\cline { 2 - 10 } & \multicolumn{2}{|c|}{ Cuento } & \multicolumn{2}{|c|}{ Carta } & \multicolumn{2}{c|}{ Noticia } & \multicolumn{2}{c|}{} \\
\cline { 2 - 10 } & $\mathrm{n}$ & $\%$ & $\mathrm{n}$ & $\%$ & $\mathrm{n}$ & $\%$ & $\mathrm{n}$ & $\%$ \\
\hline Carencia de tildes & 1046 & $58 \%$ & 205 & $35 \%$ & 291 & $39 \%$ & 1542 & $49,2 \%$ \\
\hline Omisión de letras o sílabas & 100 & $6 \%$ & 89 & $15 \%$ & 77 & $10 \%$ & 266 & $8,5 \%$ \\
\hline Uso de b/v & 145 & $8 \%$ & 33 & $6 \%$ & 45 & $6 \%$ & 223 & $7,1 \%$ \\
\hline Uso de h & 100 & $6 \%$ & 31 & $5 \%$ & 83 & $11 \%$ & 214 & $6,8 \%$ \\
\hline Uso de s/c/z & 128 & $7 \%$ & 43 & $7 \%$ & 60 & $8 \%$ & 231 & $7,4 \%$ \\
\hline Hiposegmentación & 74 & $4 \%$ & 54 & $9 \%$ & 33 & $4 \%$ & 161 & $5,1 \%$ \\
\hline Otros tipos de errores & 212 & $12 \%$ & 125 & $22 \%$ & 162 & $22 \%$ & 499 & $15,9 \%$ \\
\hline Total & 1805 & $100 \%$ & 580 & $100 \%$ & 751 & $100 \%$ & 3136 & $100 \%$ \\
\hline
\end{tabular}

Fuente: elaboración propia.

$\mathrm{Al}$ analizar los resultados por género textual, podemos ver en la Tabla 4 que en el cuento, más de la mitad de los errores corresponde a carencia de tilde, en tanto que en la carta y en la noticia, el porcentaje es más bajo. El porcentaje de errores de omisión de letras o sílabas se distribuye de distinta manera, ya que es mayor en la carta y menor en el cuento, en tanto que el uso erróneo de grafías (b/v, h, s/c/z) es mayor en la noticia y menor en la carta.

\subsection{ANÁLISIS DE CORRELACIONES POR GÉNERO TEXTUAL}

Los análisis comparativos presentados a continuación se elaboraron a partir de un Coeficiente de Error Ortográfico (CEO = No de errores x $100 / \mathrm{N}^{\mathrm{o}}$ palabras escritas) sobre una muestra de 231 textos, considerando solo los 77 estudiantes que escribieron los tres géneros. En la Tabla 5 es posible apreciar que globalmente, el CEO general del estudio es de 18 puntos. Si se analizan los resultados obtenidos por género escrito, es posible ver que en los cuentos y las noticias los alumnos presentan un CEO mayor y semejante entre sí, mientras que en las cartas los alumnos obtienen un CEO significativamente menor.

Tabla 5. Promedio de Coeficiente de Error Ortográfico (CEO)

\begin{tabular}{|c|c|c|c|c|c|c|c|c|c|c|c|c|c|c|c|}
\hline \multicolumn{3}{|c|}{ Cuento } & \multicolumn{3}{c|}{ Carta } & \multicolumn{3}{c|}{ Noticia } & \multicolumn{3}{c|}{ Total } \\
\hline \multicolumn{3}{|c|}{$(\mathrm{n}=77)$} & \multicolumn{3}{c|}{$(\mathrm{n}=77)$} & \multicolumn{3}{c|}{$(\mathrm{n}=77)$} & \multicolumn{3}{c|}{ (n=231) } \\
\hline Media & Min & Máx & DE & Media & Min & Máx & DE & Media & Min & Máx & DE & Media & Min & Máx & DE \\
\hline $\mathbf{2 0 , 3}$ & 0,8 & 53,7 & 10 & $\mathbf{1 3 , 5}$ & 1,5 & 63,6 & 9,8 & $\mathbf{1 8 , 8}$ & 2,2 & 50 & 10,7 & $\mathbf{1 8}$ & 2,3 & 45,5 & 8,4 \\
\hline
\end{tabular}

Diferencias*: $($ Cuento $=$ Noticia $)>$ Carta

*Diferencias son significativas si $p<0,05$ 
En las tablas siguientes, 6 a 8, se presentan los resultados de las correlaciones entre las variables CEO, número de palabras, calidad del texto, NSE y sexo, obtenidos para cada género textual.

Tabla 6. Correlación entre variables en cuentos $(\mathrm{n}=77)$

\begin{tabular}{|c|l|c|c|c|c|c|}
\hline & & 1 & 2 & 3 & 4 & 5 \\
\hline $\mathbf{1}$ & CEO & 1 &,$- 284^{*}$ &,$- 370^{* *}$ &,$- 408^{* *}$ &,$- 385^{* *}$ \\
\hline $\mathbf{2}$ & $\mathrm{N}^{\circ}$ de palabras & & 1 &, $532^{* *}$ &, 048 &, 211 \\
\hline $\mathbf{3}$ & Calidad del texto & & & 1 &, $397^{* *}$ &, 221 \\
\hline $\mathbf{4}$ & NSE & & & & 1 &,- 052 \\
\hline $\mathbf{5}$ & Sexo & & & & & 1 \\
\hline
\end{tabular}

$* p<.05 * * p<.01$

Fuente: elaboración propia.

Las correlaciones de la variable CEO indican que este es menor cuando hay un mayor número de palabras escritas, una mayor calidad del texto, y un NSE más alto. Además, las mujeres presentan un menor CEO que los hombres.

Respecto de las palabras escritas, mientras mayor es su número, mayor es la calidad de los textos. Otra correlación significativa es la que se da entre una mayor calidad de los textos escritos y los NSE más altos.

Tabla 7. Correlación entre variables en cartas $(\mathrm{n}=77)$

\begin{tabular}{|c|l|c|c|c|c|c|}
\hline & & 1 & 2 & 3 & 4 & 5 \\
\hline $\mathbf{1}$ & CEO & 1 &,- 218 &,$- 301^{* *}$ &,$- 307^{* *}$ &,- 186 \\
\hline $\mathbf{2}$ & $\mathrm{N}^{\circ}$ de palabras & & 1 &, $316^{* *}$ &, 074 &, 100 \\
\hline $\mathbf{3}$ & Calidad del texto & & & 1 &, $277^{*}$ &, $322^{* *}$ \\
\hline $\mathbf{4}$ & NSE & & & & 1 &,- 052 \\
\hline $\mathbf{5}$ & Sexo & & & & & 1 \\
\hline
\end{tabular}

$* p<.05 * * p<.01$

Fuente: elaboración propia.

Para el caso de las cartas, el CEO también correlaciona inversamente con la calidad de los textos y el NSE. Es menor el CEO en los textos que presentan una mejor calidad y en el NSE más altos.

A su vez, el mayor número de palabras escritas correlaciona positivamente con una mayor calidad de los textos. Adicionalmente, se repite la correlación entre calidad de los textos en los NSE más altos. Cabe destacar que en este género se observó una correlación positiva entre el índice de calidad y el sexo femenino. 
Tabla 8. Correlación entre variables en noticias $(\mathrm{n}=77)$

\begin{tabular}{|c|c|c|c|c|c|c|}
\hline & & 1 & 2 & 3 & 4 & 5 \\
\hline $\mathbf{1}$ & CEO & 1 &,- 176 &,$- 345^{* *}$ &,$- 446^{* *}$ &,- 217 \\
\hline $\mathbf{2}$ & $\mathrm{N}^{\circ}$ de palabras & & 1 &, 131 &,- 003 &, 201 \\
\hline $\mathbf{3}$ & Calidad del texto & & & 1 &, $379^{* *}$ &, 138 \\
\hline $\mathbf{4}$ & NSE & & & & 1 &,- 052 \\
\hline $\mathbf{5}$ & Sexo & & & & & 1 \\
\hline
\end{tabular}

$* p<.05 * * p<.01$

Fuente: elaboración propia.

En las noticias, el CEO correlaciona inversamente tanto con la calidad del texto como con el NSE. A su vez, el NSE correlaciona positivamente con la calidad del texto. Dicho de otra manera, mientras menor es el NSE, menor es la calidad del texto y mayor el CEO.

En suma, en el caso de las noticias, al igual que en los cuentos y en las cartas, es menor el CEO cuando la calidad de los textos es mayor y en los niveles NSE más altos. Lo mismo sucede respecto de la calidad de los textos, pues esta es mayor en los niveles socioeconómicos más altos.

\subsection{ESTIMACIÓN DE ERRORES SISTEMÁTICOS Y ERRORES AZAROSOS}

En la muestra se detectaron 3136 errores ortográficos. Sin embargo, no se sabe con claridad qué porcentaje de estos errores se debe al desconocimiento o simplemente al olvido de una tilde o una grafía; en palabras de Ruiz (2009), a un error (sistemático) o a una equivocación (azarosa). Este hecho es importante, pues los errores sistemáticos denotan un problema en la competencia; mientras que los azarosos, una carencia de estrategias de revisión. Con el fin de estimar hasta qué punto los errores de los estudiantes podrían ser azarosos, fueron rastreados en la muestra los casos en que una palabra escrita incorrectamente por un niño fue también escrita correctamente al menos una vez por el mismo niño. De esta manera, se encontró que los 101 niños de la muestra cometieron al menos un error ortográfico, pero 84 de ellos dieron muestra de haber escrito correctamente al menos una de las palabras en las que cometieron errores. Ejemplos de ello se pueden apreciar en los siguientes textos. La Figura 1, muestra cómo la palabra "delfín" fue escrita con las grafías que corresponde una vez, mientras que en la segunda aparición se lee "delfil". 
Figura 1. Ejemplo de equivocación de "n" por "l"

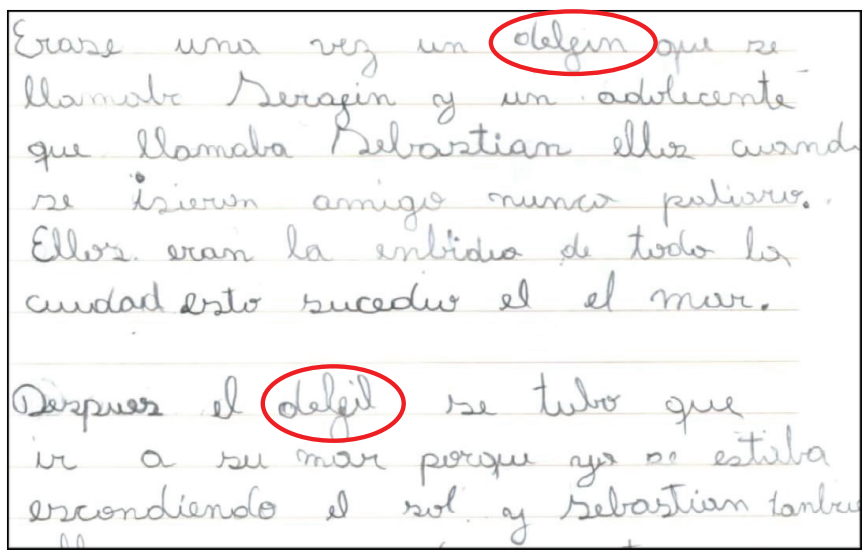

La Figura 2, en tanto, muestra un caso en que el niño escribe la palabra "papás" con acento y posteriormente lo omite, algo que también podría interpretarse como una equivocación.

Figura 2. Ejemplo de equivocación por omisión de tilde

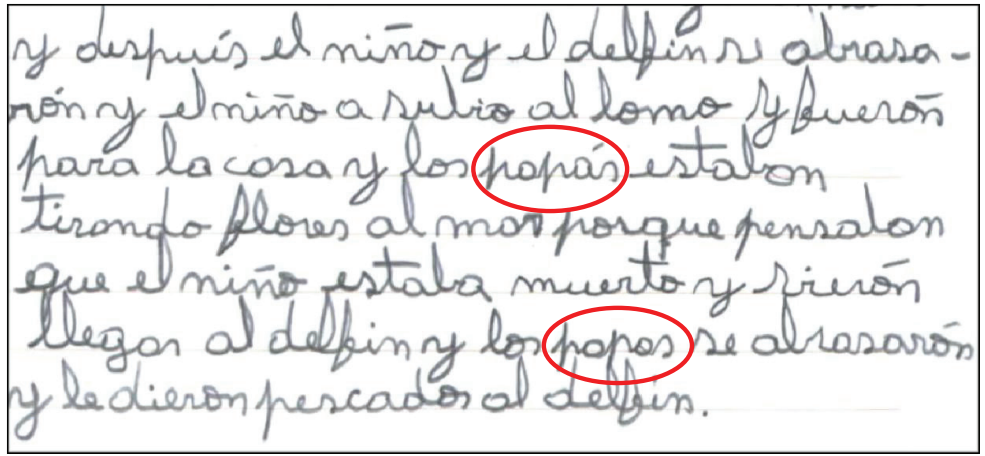

Estos datos sugieren que es probable que en muchos casos los niños conozcan la forma correcta de la palabra, pero se equivoquen mientras escriben dada la alta demanda cognitiva de la tarea.

En tanto, solo 17 de los 101 niños en la muestra son consistentes en los errores que cometen, como se muestra en el siguiente ejemplo, donde "huesos" fue escrito como "guesos" las tres veces que apareció en el texto (Figura 3). La Figura 4 muestra el mismo caso con la palabra "por favor" escrita consistentemente como "porfabor" a lo largo de todo el texto. 
Figura 3. Ejemplo de error sistemático: "guesos"

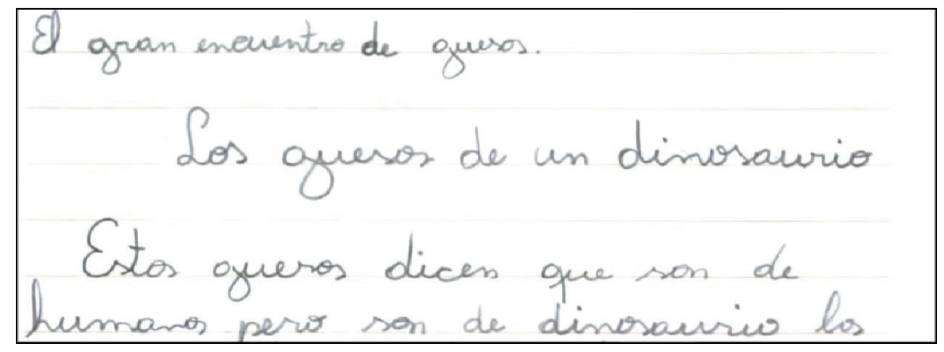

Figura 4. Ejemplo de error sistemático: "porfabor"

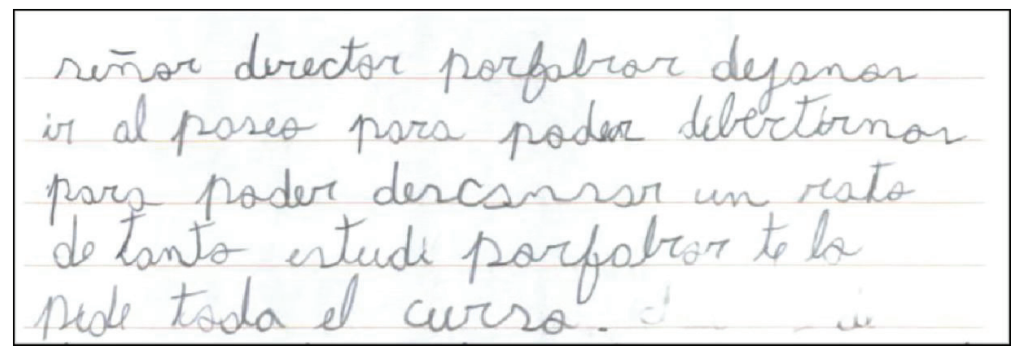

En estos casos, el error ortográfico denota que el niño desconoce la ortografía de la palabra, para lo que se requiere intervención didáctica explícita (mostrar cómo se escribe) más allá de las meras estrategias de revisión.

\section{CONCLUSIONES}

Uno de los principales hallazgos de esta aproximación al desempeño ortográfico es que los estudiantes chilenos de cuarto grado escriben en promedio $91 \%$ de palabras correctas en sus producciones textuales, con una ligera variación según el género que se considere. Este resultado es consistente con los estudios realizados anteriormente tanto en Chile (Molina, 2012; Sotomayor et al., 2013), como en América Latina (Atorresi et al., 2010). La importancia de cuantificar el error reside en remover los mitos respecto a la mala ortografía de los estudiantes, lo que permite concentrar los esfuerzos educativos en este repertorio acotado, para focalizar la enseñanza en aquello que resulta más difícil. En un estudio previo (Sotomayor et al., 2013) que analizó textos de nivel socioeconómico bajo en una situación de evaluación se encontró $84 \%$ de palabras correctamente escritas, un número alto que se acerca a lo encontrado en el presente estudio con niños de diversos sectores sociales. Este porcentaje tan bajo de errores también puede estar influido por el contexto 
de producción, pues el SIMCE es una medición educativa de gran interés social en el país, que puede implicar un mayor esfuerzo de los estudiantes, dada la expectativa percibida de dicha práctica académica (Sebba, 2007). De hecho, estas variables contextuales han sido reportadas por trabajos previos, como el estudio de Campbell Wilcox et al. (2014), en que se comparó el desempeño escrito de adolescentes en la asignatura de Lengua Materna y Ciencias Sociales en Estados Unidos. Los resultados mostraron una tasa de error significativamente menor en la clase de lengua, al comparar un promedio de 5.34 errores en Inglés con 7.14 en Ciencias, porque los estudiantes se esforzaron más en la asignatura donde se trabaja y exige la corrección en el lenguaje y la escritura legible. Con todo, los resultados son indicativos del desempeño de los estudiantes chilenos y permiten sacar conclusiones valiosas dada la representatividad de la muestra, como se presenta a continuación.

El tipo de error ortográfico más frecuente fue la carencia de tilde. El $92 \%$ de los estudiantes presentó este error al menos una vez en sus escritos. En segundo lugar, se encontraron errores relacionados con la omisión de letras y sílabas, el uso erróneo de grafías (b/v, h, s/c/z) y la hiposegmentación, presentes en más de un tercio de los estudiantes. Estos mismos resultados mostraron los estudios realizados en Chile por Sotomayor et al. (2013) y en México por Backhoff et al. (2008). De acuerdo con esta evidencia, estos errores estarían relacionados con las dificultades propias de los sistemas alfabéticos, en los que no existe univocidad entre grafemas y sonidos. La omisión de letras también puede vincularse a la etapa de desarrollo y las múltiples demandas cognitivas de la producción textual, en un momento en que el código no ha sido por completo internalizado. Por último, los problemas de hiposegmentación están asociados a la complejidad que representa para los niños pequeños alcanzar una conciencia de las palabras por el continuo sonoro que caracteriza al lenguaje oral. En el estudio fue habitual que el acoplamiento se produjera entre palabras funcionales y locuciones (artículos, conjunciones, preposiciones) y palabras de contenido (sustantivos y verbos), por ejemplo, 'ala mar', 'allorar', 'enverdad', etc. En todos estos casos, los errores observados sugieren la necesidad de trabajar en el aula estrategias de revisión de los textos, focalizadas en fenómenos de omisión de grafías, uso de b/v, h, s/c/z y casos prototípicos de hiposegmentación.

Por otra parte, respecto del dominio ortográfico de los alumnos, calculado por el coeficiente de error ortográfico (CEO), el análisis mostró 18 puntos para la totalidad de los escritos, sin embargo, tanto en el cuento como en la noticia, el CEO fue significativamente mayor que en las cartas. Al respecto, vale hacer notar que, en los tres géneros solicitados, difería el grado de referencia a la situación y al potencial lector concreto. Para el cuento, no se explicitaba la situación, la audiencia ni el propósito; para la carta, se explicitaba la situación (paseo de curso), el propósito (pedir permiso) y un destinatario (director/a); para la noticia, se explicitaban solo la situación (hallazgo arqueológico en el colegio) y una audiencia, aunque difusa (diario mural). La principal conclusión de este resultado radica en la importancia de desarrollar en el aula tareas en las que se explicite una situación retórica, con género y un destinatario claramente identificable (Sotomayor, Ávila \& Jéldrez, 2015). La escritura requiere necesariamente estos factores para mediar una representación de la tarea, y la generación de metas y expectativas por parte del escritor (Scardamalia \& Bereiter, 1992). Cuentos y noticias obtuvieron similares CEO, 20.3 y 18.8, respectivamente, mientras que en las cartas, el CEO resultó significativamente más bajo, lo que puede relacionarse con la explicitación de los elementos de la situación, en consonancia con la propuesta de 
Camps et al. (2007), para quienes la ortografía tiene sentido en la medida que les resulta necesaria a los estudiantes en situaciones comunicativas.

Este resultado es también indicativo de la importancia de considerar factores pragmáticos en el desempeño ortográfico, como lo sugieren resultados de Campbell Wilcox et al. (2014). La carta de solicitud no solo fue el único de los tres géneros propuestos en las tareas del SIMCE 2008 que describía un lector claramente identificable por los estudiantes, sino que este destinatario era nada menos que el director o directora del establecimiento. Se trata de una figura que representa la autoridad educativa, lo que sugiere hasta qué punto factores como el poder relativo de los participantes y la distancia social (Brown \& Levinson, 2004) impactan en el desempeño escrito.

Una correlación interesante se encontró en los cuentos, en los que la cantidad de palabras escritas y el CEO correlacionaron negativamente: a mayor cantidad de palabras escritas, menor CEO. Este resultado confirma los planteamientos de Berninger et al. (1992) respecto al rol que las habilidades de bajo nivel, como la codificación ortográfica, juegan en el subcomponente de transcripción, lo que sugiere que una mayor cantidad de palabras es una consecuencia de un mayor dominio ortográfico en este nivel del desarrollo. Sin embargo, esto no se observó de igual modo en cartas de solicitud y noticias, diferencia que puede deberse al menor conocimiento de los niños de educación básica de estos géneros, debido al currículum vigente al momento de la prueba y a la mayor familiaridad del cuento. Según McCutchen (2011), el desconocimiento del género implica mayores demandas cognitivas que pueden afectar la fluidez. Esta hipótesis resulta sugerente y releva la necesidad de estudios que indaguen la interrelación de las demandas cognitivas asociadas a habilidades de bajo nivel, de alto nivel y conocimientos relevantes sobre la escritura (como los géneros) que puedan influir en la calidad de los textos.

Otro resultado de interés muestra que las mujeres obtienen un menor índice de error ortográfico en el cuento y un mayor índice de calidad en las cartas. Este mejor desempeño en dos de las tres variables analizadas muestra la necesidad de realizar futuras investigaciones que identifiquen aquellos factores de la enseñanza o del aprendizaje que están produciendo estas diferencias, con el fin de propiciar entre los docentes prácticas de escritura, dentro y fuera del aula de lenguaje, equitativas entre niños y niñas.

Al correlacionar el CEO con nivel socioeconómico pudo apreciarse que mientras más alto es el nivel socioeconómico de los alumnos, menor es el CEO. Asimismo, al considerar el índice de calidad del texto, los resultados fueron consistentes en mostrar que en los tres géneros analizados el puntaje de calidad obtenido se correlaciona en forma inversa con el CEO y directa con el nivel socioeconómico. Es decir, la mayor calidad textual implica un menor error ortográfico y se da en mayor medida en los NSE más altos. Estos resultados corroboran la desigualdad de los aprendizajes en las aulas chilenas. Por ello, es indispensable mantener las políticas de priorización o discriminación positiva llevadas adelante en las últimas décadas en Chile. Tanto los resultados asociados a NSE como a sexo confirman la misma tendencia que muestran las pruebas nacionales de lectura y escritura en las que se aprecian diferencias significativas a favor de las mujeres y de los niveles socioeconómicos más altos (Ministerio de Educación de Chile, 2009).

Por último, con respecto a la diferencia entre errores sistemáticos y azarosos, una estimación hecha al comparar los errores cometidos por un niño con el total de veces en que la palabra fue escrita por el mismo niño, permitió detectar 84 casos en los que los niños podían dar evidencia de conocer la forma correcta de al menos uno de sus 
errores. Este resultado refuerza la importancia de trabajar las estrategias de corrección de manera constante, ya que algunos niños podrían mejorar significativamente su desempeño ortográfico si incorporasen la revisión a sus rutinas de producción escrita.

En síntesis, el estudio realizado confirma lo propuesto por algunos autores (Backhoff et al., 2008; Campbell Wilcox et al., 2014; Tuana, 1980) respecto de que la enseñanza de la ortografía debe enfocarse más en el análisis y reflexión sobre los errores ortográficos, que en el aprendizaje de todas las normas. Ello porque, como se ha demostrado en esta investigación, los errores están concentrados en algunas palabras y fenómenos. Al mismo tiempo, este estudio reafirma la importancia de practicar de manera sistemática la escritura en el aula y desarrollar la enseñanza ortográfica a partir de la escritura de textos en situaciones retóricas bien definidas, en lo posible con lectores reales, de manera de crear la necesidad y la motivación para desarrollar esta habilidad. Por último, es importante reforzar estas habilidades, que son tan importantes para el desarrollo de habilidades superiores de la composición, en particular en los grupos de NSE bajo, de manera de acortar las brechas de desempeño que resultan frecuentes en nuestro sistema de educación.

\section{REFERENCIAS BIBLIOGRÁFICAS}

Atorresi, A., Bengochea, R., Bogoya, D., Burga, A., Castro, M., García, F., Jurado, F. Martínez, R., \& Pardo, C. (2010). Escritura. Un estudio de las habilidades de los estudiantes de América Latina y el Caribe. Santiago: Unesco-Orealc.

Backhoff, E., Peon, M., Andrade, E., \& Rivera, S. (2008). La ortografía de los estudiantes de educación básica en México. México: Instituto Nacional para la Evaluación de la Educación.

Berninger, V., Yates, C., Cartwright, A., Rutberg, J., Remy, E., \& Abbott, R. (1992). Lower-level developmental skills in beginning writing. Reading and Writing, 4(3), 257-280. doi:10.1007/ BF01027151

Blanche-Benveniste, C. (1998). Estudios lingüísticos sobre la relación entre oralidad y escritura. Barcelona: Gedisa.

Brown, P., \& Levinson, S. (2004). Politeness: Some universals in language use. Cambridge: University of Cambridge Press.

Campbell Wilcox, K., Yagelski, R., \& Yu, F. (2014). The nature of error in adolescent student writing. Reading and Writing, 27(6), 1073-1094. doi:10.1007/s11145-013-9492-x

Camps, A., Milian, M., Bigas, M., Camps, M., \& Cabré, P. (2007). La enseñanza de la ortografía (5ta ed.). Barcelona: GRAO.

Condemarín, M., \& Medina, A. (2000). Evaluación de los aprendizajes. Santiago de Chile: MINEDUC. doi:10.1017/CBO9781107415324.004

Díaz, M. (2010). Beneficios de la revisión en la didáctica de la ortografía. Bordón, 62, 65-79.

Dos Santos, M. J., \& Barrera, S. D. (2012). Relaçáo entre conhecimento explícito da ortografia e desempenho ortográfico. Psicologia Escolar e Educacional, 16(2), 257-263. doi:10.1590/ S1413-85572012000200008

Ferreiro, E. (2007). Las inscripciones de la escritura. Conferencia pronunciada el día 15 de marzo en la Universidad de La Plata, durante el acto de entrega de la designación Doctora Honoris Causa por esa institución.

Flotts, P., Manzi, J., Jiménez, D., Abarzúa, A., Cayumán, C., \& García, M. (2015). Informe de Resultados TERCE. Santiago: OREALC/UNESCO.

Gee, J. P. (2004). Social Linguistics And Literacies: Ideology in Discourses (2nd ed.). London: Routledge Falmer. 
Kaufman, A. M. (2005). Cómo enseñar, corregir y evaluar la ortografía de nuestros alumnos... y no morir en el intento. Lectura Y Vida, 26(3), 6-21.

Mccutchen, D. (2011). From novice to expert : Implications of language skills and writing-relevant knowledge for memory during the development of writing skill. Journal of Research on Writing, 3, 51-68.

Ministerio de Educación de Chile. (2009). Informe de resultados de escritura $4^{\circ} 2008$. Santiago de Chile: SIMCE, Unidad de Currículum y Evaluación.

Molina, D. (2012). Habilidades de Escritura en niños de Educación Básica: Caracterización del desempeño ortográfico en narraciones de escolares de $3^{\circ}, 5^{\circ}$ y $7^{\circ}$ año de educación básica pertenecientes a escuelas municipales rurales de Constitución y Empedrado (Tesis para optar al grado de Magíster en Lingüística, mención Lengua Española). Universidad de Chile, Santiago.

Morales, O., \& Hernández, L. (2004). Estudio descriptivo del uso de la ortografía de los estudiantes universitarios de nuevo ingreso. Kaleidoscopio, 2, 151-159.

Real Academia de la Lengua Española. (2010). Ortografía de la lengua española. Madrid: Espasa.

Ruiz, M. (2009). Evaluación de la lengua escrita y dependencia de lo literal. Barcelona: GRAÓ.

Sánchez, D. (2010). El análisis de errores ortográficos de estudiantes filipinos en el aprendizaje de español como LE y su aplicación didáctica. I Congreso de Español como Lengua Extranjera en Asia-Pacífico, 106-126. Recuperado desde http://cvc.cervantes.es/ensenanza/biblioteca_ele/ publicaciones_centros/pdf/manila_2009/09_investigaciones_02.pdf

Sánchez, V., Moyano, V., \& Borzone, A. (2011). Demandas cognitivas de la escritura: comparación de dos situaciones de producción. Estudios pedagógicos, 37, 227-236.

Scardamalia, M., \& Bereiter, Y. C. (1992). Dos modelos explicativos de los procesos de composición escrita. Infancia y Aprendizaje, 58, 43-64.

Sebba, M. (2007). Spelling and society. Cambridge: Cambridge University Press.

Smith-Lock, K. M., Nickels, L., \& Mortensen, L. (2009). Story writing skills of adults with a history language-impairment. Reading and Writing, 22(6), 713-734. doi:10.1007/s11145-008-9138-6

Sotomayor, C., Ávila, N., \& Jéldrez, E. (Coords.). (2015). Rúbricas y otras herramientas para desarrollar la escritura en el aula. Santiago de Chile: Santillana.

Sotomayor, C., Molina, D., Bedwell, P., \& Hernández, C. (2013). Caracterización de problemas ortográficos recurrentes en alumnos de escuelas municipales chilenas de $3^{\circ}, 5^{\circ}$ y $7^{\circ}$ básico. Revista Signos, 46(81), 105-131. doi:10.4067/S0718-09342013000100005

Tuana, E. (1980). Estudio comparativo de la ortografía en niños normales y niños con dificultades de aprendizaje. Lectura y Vida, 1(1), 16-21.

Tuana, E., Carbonell, M., \& Lluch, E. (1980). Diez años de investigaciones ortográficas. Lectura y Vida, 1(2), 16-19. 